\title{
Voice Outcome after Gore-Tex Medialization Thyroplasty
}

\author{
Ismail Elnashar ${ }^{1}$ Mohammad El-Anwar ${ }^{1} \quad$ Hazem Amer $^{1} \quad$ Amal Quriba $^{1}$ \\ ${ }^{1}$ Department of Otorhinolaryngology-Head and Neck Surgery, \\ Zagazig University, Zagazig, Egypt \\ Int Arch Otorhinolaryngol 2015;19:248-254. \\ Address for correspondence Mohammad Waheed El-Anwar, MD, \\ Department of Otorhinolaryngology Head and Neck Surgery, Faculty \\ of Medicine, Zagazig University, Zagazig 0020552309843, Egypt \\ (e-mail: mwenteg@yahoo.com).
}

\begin{abstract}
Keywords

- thyroplasty

- voice

- Gore-Tex
\end{abstract}

\section{Introduction}

Glottic incompetence (GI) can be defined as incomplete glottic closure during phonation. It is a common finding in patients with vocal fold immobility, paresis, atrophy, and scar. ${ }^{1}$

Unilateral vocal fold paralysis (UVFP) is the most common cause of GI and usually presents with dysphonia and possibly with severe symptoms such as aspiration, poor coughing effort, or pneumonia, which may be life-threatening in debilitated

received

October 22, 2014

accepted

November 18, 2014

published online

February 26, 2015
DOI http://dx.doi.org/

10.1055/s-0034-1397339. ISSN 1809-9777. patients. ${ }^{2-4}$ It was estimated that 23 to $53 \%$ of patients with UVFP demonstrated evidence of aspiration on videofluoroscopic examination. ${ }^{3-5}$ If there is no recovery or if there is inadequate compensation after UVFP, procedures aiming to restore glottic competence include permanent and temporary vocal fold injections or laryngeal framework surgery, such as type 1 thyroplasty. ${ }^{6}$

Type I thyroplasty, with or without adjunctive arytenoids procedures, is widely considered the surgical management of choice for glottic insufficiency resulting from vocal fold

Copyright $\odot 2015$ by Thieme Publicações License terms Ltda, Rio de Janeiro, Brazil

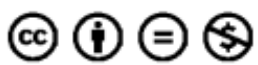


immobility. Medialization using laryngeal implants has been shown to improve voice handicap index and perceptual voice measures as well as acoustic parameters of the voice. ${ }^{7-9}$ Both Silastic and Gore-Tex (both Gore and Associates, Newark, Delaware, United States) thyroplasty implants have been shown to provide excellent long-term surgical rehabilitation of paralytic dysphonia. ${ }^{10,11}$ The ideal surgical management of GI in patients with mobile vocal folds, however, is far less well established.

Gore-Tex, expanded polytetrafluoroethylene, was developed by Gore and Associates in the late 1960s. The first application was in general vascular surgery, and the first vessel implants were described in $1971 .{ }^{12}$ Since that time, the material has been described as a semiporous material with excellent tissue integration, minimal tissue inflammation, and low rates of extrusion and is easily removed when necessary. $^{13,14}$

Gore-Tex medialization laryngoplasty was first described by McCulloch and Hoffman in $1998 .^{10}$ The technique is fast and not technically demanding because the implant is simply layered into the paraglottic region and does not need to be carved. Medialization is reached in an incremental manner (inserting or removing portions of the Gore-Tex strip) until satisfactory vocal outcome is achieved, allowing fine intraoperative adjustments that do not involve removal and replacement of the entire implant. ${ }^{15}$

Although medialization thyroplasty utilizing Gore-Tex was discussed in the literature, few reports assessed the voice quality after the surgery, and even these did not use full assessment protocol. ${ }^{1,2,5}$ The aim of this study was to assess the improvement in voice quality after Gore-Tex medialization thyroplasty in patients with uncompensated GI using a full assessment protocol and comparing the immediate postoperative results to later results.

\section{Methods}

This study was conducted in between June 2007 and October 2013. The study included 11 patients who presented with insufficient glottic closure that failed to compensate at least 1 year after injury. Three patients were previously exposed to vocal fold injection at least 6 months prior to medialization, with poor results. The etiology of glottic insufficiency was vocal fold paralysis in 10 patients (postthyroidectomy in 5 patients, idiopathic in 2, after spine surgery in 2, carotid body tumor in 1 , and after cordectomy in 1). All the patients were operated using type 1 thyroplasty utilizing Gore-Tex. Preoperatively, all the patients had voice complaints in the form of breathy dysphonia, increased phonation effort, and vocal fatigue. All of them had glottic phonatory gap more than $3 \mathrm{~mm}$ at its widest point. Informed written consent was obtained from all included subjects. The patients were subjected to preoperative voice assessment protocol by the phoniatrician, who was blinded to the state of the patient (either preoperative or postoperative). ${ }^{16}$ This protocol passed through the following levels: elementary diagnostic procedures, clinical diagnostic aids, and additional instrumental measures.

\section{Elementary Diagnostic Procedures}

Diagnostic procedures included auditory perceptual assessment of the patient's voice as a subjective evaluation of the voice with careful laryngeal examination. The presence or absence of vocal fold visible mobility was determined, along with its side and degree (normal or restricted) and the direction of restriction (abductor or adductor), to confirm the diagnosis. The phonatory gap (gap between vocal folds during average phonation) and respiratory chink (maximal glottic gap during respiration) were determined. They were measured in millimeters at their widest point compared with the length of the visible membranous vocal fold (proposed to be $15 \mathrm{~mm}$ in men and $10 \mathrm{~mm}$ in women). ${ }^{17}$ Glottic gap was confirmed using the method described by Kotby et al. ${ }^{17} \mathrm{~A}$ millimeter-graded ruler was photographed in focus with the same magnification used in video laryngoscopy of the glottis. The printout of the glottis was obtained from the stroboscopy system, and a printout of the ruler image was obtained as well, and the gap was then measured.

\section{Clinical Diagnostic Aids}

This level of the protocol includes laryngo-video-stroboscopy using either flexible nasofibroscope or rigid 70-degree laryngoscope, from Xion Medicals (Germany), connected to a camera in association with stroboscopic light. Phonatory gap size was confirmed during stroboscopic image with average comfortable phonation at the widest point of the vocal folds. Then documentation of the auditory perceptual assessment was done by means of high-fidelity voice recording, which included reading a standardized text, a counting task, and sustained vowel prolongation including $/ \alpha /, / \mathrm{i} /$ and $/ \mathrm{u} /$ vowels. The recordings were done in a sound-treated room to minimize environmental noise. The recorded materials for all patients were rated using the modified GRBAS (grade, roughness, breathiness, asthenia, strain) scale, ${ }^{18}$ which was confirmed by three experienced phoniatricians. This scale included 4 points ( 0 to 3; 0 for normal, 3 for severe) for determining the overall grade of dysphonia and character of voice including strained, leaky, breathy, and rough.

\section{Additional Instrumental Measures}

This level of assessment included acoustic analysis of voice using the Vocal Assessment Software from Dr Speech 4.5. The patient was asked to pronounce a prolonged $/ \alpha /$ in front of a microphone as long as possible after taking a deep inspiration. The following parameters were analyzed automatically: jitter (\%), shimmer (\%), and noise-to-harmonic ratio. Maximal phonation time (MPT) could be measured simultaneously during the same task.

Patients with mild glottic insufficiency were selected for vocal fold injection and were only operated by medialization if voice quality was unsatisfactory immediately or later after injection. Patients with moderate and severe glottic insufficiency were operated directly by Gore-Tex medialization thyroplasty. All the surgeries were done initially under local anesthesia. Postoperatively, voice assessment was repeated at 1 week, 3 months, and 6 months from the date of surgery.

Pre- and postoperative data were compared and statistical analysis is done. SPSS (Statistical Package for the Social Sciences, 
version 17; Chicago, Illinois, United States) was used to analyze the data. Data of this work were found to be homogenous using variance homogeneity test. Analysis of distribution of data revealed that parametric tests could be applied. The chi-square test was used for qualitative variables, and the analysis of variance was used for quantitative variables. Turkey honest significant difference post hoc test was used to compare individual groups. A $p$ value less than 0.05 was considered significant.

\section{Surgical Technique}

During this study, the standard Gore-Tex medialization laryngoplasty following the basic steps described McCulloch et $\mathrm{al}^{10}{ }^{10}$ with some modification, was adopted. After the administration of monitored anesthesia, $10 \%$ topical xylocaine and Oxymetazoline-soaked pledgets were placed into the nose. Topical local anesthetic spray was applied to the pharynx and a flexible nasopharyngoscope was introduced so that a clear image of the larynx was obtained. The nasopharyngoscope was fixed to a table at the patient's head and could be handled by an assistant to maintain a clear image of the larynx during the procedure. Local anesthesia, 0.5\% lidocaine $1: 200,000$ epinephrine with $0.25 \%$ bupivacaine, was then administered. A $3-\mathrm{cm}$ incision was made in a natural skin crease overlying the thyroid ala. The strap muscles were reflected laterally, and a laterally based perichondrial flap was elevated. The inferior thyrotomy as measured $5 \mathrm{~mm}$ above the inferior border of the thyroid cartilage, and a 5- to 7-mm window was then created with a no. 3 course diamond burr $5 \mathrm{~mm}$ from the midline in women and $7 \mathrm{~mm}$ from the midline in men. The inner perichondrium was identified and elevated carefully from the thyroid alae without incision. Utilizing flexible laryngoscopic guidance, the Gore-Tex was then placed with care to appropriately augment the posterior and midportions of the glottis. At the same time, the patient was asked to phonate until the best voice quality was reached. The perichondrium was draped back over the thyrotomy, and the incision was closed in layers with absorbable suture. A small drain as used and removed after 24 hours. Patients received 1 week of oral postoperative antibiotics and were discharged home the day after surgery.

\section{Results}

The study included 11 patients ( 5 men and 6 women) with ages ranging between 22 and 56 years. - Table 1 shows the demographic characteristics of the patients and the etiology of glottic incompetence.

Table 1 Descriptive data for patients included in the study before surgery

\begin{tabular}{|l|l|l|l|l|l|l|l|}
\hline Patient no. & Sex & Age (y) & Cause of paralysis & Side & $\begin{array}{l}\text { Duration till } \\
\text { surgery (mo) }\end{array}$ & $\begin{array}{l}\text { Previous inter- } \\
\text { vention }\end{array}$ & $\begin{array}{l}\text { Glottic gap size } \\
\text { (mm) }\end{array}$ \\
\hline 1 & F & 22 & After thyroidectomy & Left & 14 & None & 4 \\
\hline 2 & F & 43 & After thyroidectomy & Left & 13 & None & 5 \\
\hline 3 & M & 39 & Spine surgery & Right & 24 & $\begin{array}{l}\text { Vocal injection } \\
\text { (fat) }\end{array}$ & 4 \\
\hline 4 & F & 28 & Idiopathic & Left & 12 & None & 4 \\
\hline 5 & M & 53 & Carotid body tumor & Right & 15 & $\begin{array}{l}\text { Vocal injection } \\
\text { (fat) }\end{array}$ & 6 \\
\hline 6 & F & 38 & After thyroidectomy & Left & 12 & $\begin{array}{l}\text { Vocal injection } \\
\text { (Restylane } \mathbb{B}, \text { USA) }\end{array}$ & 5 \\
\hline 7 & M & 55 & Spine surgery & Right & 16 & None & 4 \\
\hline 8 & F & 47 & After thyroidectomy & Right & 12 & None & 4 \\
\hline 9 & M & 33 & idiopathic & Right & 13 & None & 4 \\
\hline 10 & F & 25 & After thyroidectomy & Left & 12 & None & 5 \\
\hline 11 & M & 56 & Cordectomy & Right & 18 & None & 4 \\
\hline
\end{tabular}

Table 2 Comparison between preoperative and postoperative results of the grade of dysphonia

\begin{tabular}{|c|c|c|c|c|c|c|}
\hline Grade of dysphonia & Preoperative & $1 \mathrm{wk}$ postoperative & 3 mo postoperative & 6 mo postoperative & $x^{2}$ & $p$ \\
\hline 0 & $0(0 \%)$ & $1(9 \%)$ & $2(18 \%)$ & $2(18 \%)$ & \multirow[t]{4}{*}{23.9} & \multirow[t]{4}{*}{$0.004^{a}$} \\
\hline 1 & $0(0 \%)$ & $5(45 \%)$ & $5(45 \%)$ & $4(36 \%)$ & & \\
\hline 2 & $2(18 \%)$ & $4(36 \%)$ & $3(27 \%)$ & $4(36 \%)$ & & \\
\hline 3 & $9(82 \%)$ & $1(9 \%)$ & 1 (09\%) & $1(9 \%)$ & & \\
\hline
\end{tabular}

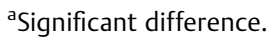


There was satisfactory improvement in the grade of dysphonia after surgery. This was confirmed by the presence of a significant difference between preoperative and all postoperative results of the grade of dysphonia (-Table 2 ). The improvement in the grade of dysphonia continued throughout the follow-up period as comparison between postoperative results of the grade of dysphonia revealed nonsignificant difference (-Table 3).

It was clear in all postoperative assessment that there was a decrease in the size of glottic gap. This was evident from the highly significant difference between preoperative and postoperative measures of glottic gap size ( - Table 4 ). Comparison between individual groups of the study confirmed the postoperative improvement (-Table 5). This improvement in the size of the glottic gap continued throughout the study period (-Table 6).

MPT was prolonged sufficiently after surgery, and this improvement continued throughout the study period. This was evident from the highly significant difference between preoperative and postoperative measurements (-Table 7) and the nonsignificant difference between postoperative measurements (-Table 8 ). These results were confirmed by comparison between individual groups (-Table $\mathbf{9}$ ).

Assessment of the preoperative acoustic analysis was impossible in all the patients due to the severe change in voice, which was detected by the software as out of range signals. Immediately after surgery, acoustic signals could be detected and calculated. This means that there was improvement of voice after surgery, and this improvement continued until the end of the study.

The surgery was done under local anesthesia in all patients except one patient (no. 6), who was converted to general anesthesia due to severe irritability. Five patients developed minor postoperative complications: three with wound ecchymosis and two with vocal fold hematoma. All of them recovered within a few days without any intervention. None of the patients developed stridor or shortness of breath necessitating tracheotomy, and there was no implant extrusion in any patient during the study period.

\section{Discussion}

The laryngeal framework surgery medialization timeline began in 1915 with Payer and has had many recent important and landmark contributions from Isshiki and others. ${ }^{19-21}$

Relatively small glottic gaps between membranous vocal folds are generally accepted to be more appropriate for injection laryngoplasty, whereas larger glottic gaps with asymptomatic posterior glottic chinks are found to be ideal for medialization thyroplasty. ${ }^{15}$ In our study, glottic phonatory gaps $\leq 3 \mathrm{~mm}$ were excluded from study. Three of the

Table 3 Comparison between different postoperative results of the grade of dysphonia

\begin{tabular}{|c|c|c|c|c|}
\hline 1 wk postoperative & 3 mo postoperative & 6 mo postoperative & $x^{2}$ & $p$ \\
\hline $1(9 \%)$ & $2(18 \%)$ & $2(18 \%)$ & \multirow[t]{4}{*}{0.725} & \multirow[t]{4}{*}{0.99} \\
\hline $5(45 \%)$ & $5(45 \%)$ & $4(36 \%)$ & & \\
\hline $4(36 \%)$ & $3(27 \%)$ & $4(36 \%)$ & & \\
\hline $1(9 \%)$ & 1 (09\%) & $1(9 \%)$ & & \\
\hline
\end{tabular}

Table 4 Comparison between preoperative and postoperative results of glottic phonatory gap

\begin{tabular}{|l|l|l|l|l|l|l|}
\hline & Preoperative & $\mathbf{1}$ wk postoperative & $\mathbf{3}$ mo postoperative & $\mathbf{6}$ mo postoperative & $\mathbf{F}$ & $\boldsymbol{p}$ \\
\hline $\begin{array}{l}\text { Size of glottic } \\
\text { gap (mm) }\end{array}$ & $4.5 \pm 0.7$ & $1.1 \pm 0.6$ & $1.5 \pm 0.9$ & $1.7 \pm 0.8$ & 46.16 & $<0.001^{\mathrm{a}}$ \\
\hline
\end{tabular}

Note: Results are mean \pm standard deviation. F: one-way analysis of variance.

a Highly significant difference.

Table 5 Post hoc test for results of comparison between phonatory gap sizes

\begin{tabular}{|l|l|l|}
\hline Comparison & Significant? $(\boldsymbol{p}<\mathbf{0 . 0 5}$ ?) & $\boldsymbol{t}$ \\
\hline 1. Preoperative-1 wk postoperative & Yes & 10.515 \\
\hline 2. Preoperative-3 mo postoperative & Yes & 9.278 \\
\hline 3. Preoperative-6 mo postoperative & Yes & 8.660 \\
\hline 4. 1 wk postoperative-3 mo postoperative & No & 1.237 \\
\hline 5. 1 wk postoperative-6 mo postoperative & No & 1.856 \\
\hline 6. 2.43 mo postoperative-6 mo postoperative & No & 0.619 \\
\hline
\end{tabular}


Table 6 Comparison between postoperative results of glottic phonatory gap size

\begin{tabular}{|l|l|l|l|l|}
\hline $\mathbf{1}$ wk postoperative & $\mathbf{3}$ mo postoperative & $\mathbf{6}$ mo postoperative & $\mathbf{F}$ & $\boldsymbol{P}$ \\
\hline $1.1 \pm 0.6$ & $1.5 \pm 0.9$ & $1.7 \pm 0.8$ & 1.7 & 0.2 \\
\hline
\end{tabular}

Note: Results are mean \pm standard deviation. F: one-way analysis of variance.

Table 7 Comparison between preoperative and postoperative results of acoustic analysis and MPT

\begin{tabular}{|c|c|c|c|c|c|c|}
\hline $\begin{array}{l}\text { Acoustic analysis } \\
\text { and MPT }\end{array}$ & Preoperative & 1 wk postoperative & 3 mo postoperative & 6 mo postoperative & $\mathrm{F}$ & $p$ \\
\hline F0 & Out of range & $\begin{array}{l}\text { Male: } 110 \pm 15 \\
\text { Female: } 249 \pm 23\end{array}$ & $\begin{array}{l}\text { Male: } 112 \pm 12 \\
\text { Female: } 251 \pm 22\end{array}$ & $\begin{array}{l}\text { Male: } 109 \pm 19 \\
\text { Female: } 247 \pm 24\end{array}$ & $\begin{array}{l}0.105 \\
0.083\end{array}$ & $\begin{array}{l}0.9 \\
0.9\end{array}$ \\
\hline Jitter (\%) & Out of range & $0.9 \pm 0.5$ & $1.1 \pm 0.3$ & $1.2 \pm 0.3$ & 1.79 & 0.18 \\
\hline Shimmer (\%) & Out of range & $2.5 \pm 1.2$ & $2.3 \pm 1.4$ & $2.1 \pm 1.3$ & 0.25 & 0.77 \\
\hline F0 tremor & Out of range & $0.7 \pm 0.5$ & $0.9 \pm 0.1$ & $0.9 \pm 0.2$ & 1.47 & 0.25 \\
\hline HNR & Out of range & $20 \pm 2.4$ & $18 \pm 3.1$ & $21 \pm 5$ & 1.9 & 0.17 \\
\hline MPT & $7 \pm 5$ & $21 \pm 7$ & $22 \pm 5$ & $20 \pm 4$ & 19 & $<0.001^{\mathrm{a}}$ \\
\hline
\end{tabular}

Abbreviations: MPT, maximum phonation time; NHR, noise to harmonic ratio.

Note: Results are mean \pm standard deviation. F: one-way analysis of variance.

${ }^{a}$ Highly significant difference.

Table 8 Comparison between postoperative results of maximum phonation time (MPT)

\begin{tabular}{|l|l|l|l|l|l|}
\hline & 1 wk postoperative & 3 mo postoperative & 6 mo postoperative & F & $p$ \\
\hline MPT & $21 \pm 7$ & $22 \pm 5$ & $20 \pm 4$ & 0.367 & 0.69 \\
\hline
\end{tabular}

Note: Results are mean \pm standard deviation. F: one-way analysis of variance.

Table 9 Post-hoc test for results of comparison between MPT

\begin{tabular}{|l|l|l|}
\hline Comparison & Significant? $(\boldsymbol{p}<\mathbf{0 . 0 5}$ ?) & $\boldsymbol{t}$ \\
\hline 1. Preoperative-1 wk postoperative & Yes & 6.123 \\
\hline 2. Preoperative-3 mo postoperative & Yes & 6.561 \\
\hline 3. Preoperative-6 mo postoperative & Yes & 5.686 \\
\hline 4. 1 wk postoperative-3 mo postoperative & No & 0.437 \\
\hline 5. 1 wk postoperative-6 mo postoperative & No & 0.437 \\
\hline 6. 3 mo postoperative-6 mo postoperative & No & 0.875 \\
\hline
\end{tabular}

included patients were previously exposed to vocal fold injection at least 6 months prior to medialization, with poor results. The poor results for injection of those patients are expected to be due to faulty selection of the surgical technique, as they had gap size more than $3 \mathrm{~mm}$. Another reason may be atrophy and scarring of the vocal cord after injection.

Despite the great advantages of Gore-Tex medialization laryngoplasty, some studies reported delayed extrusion of the Gore-Tex prosthesis. ${ }^{22,23}$ In these studies, the inner perichondrium was incised or removed. We did not incise the inner perichondrium, and we did not have any cases of implant extrusion. The importance of preservation of the inner perichondrium of the thyroid cartilage was also emphasized by other authors. ${ }^{24}$ They recommend that one should proceed very carefully and cautiously while dissecting the inner perichondrium, otherwise hemorrhage may develop, and in performing hemostasis, the coagulation may damage the delicate structure.

The authors of this work agree with Bihari et al that when performing thyroplasties using the techniques reported by Isshiki et al. ${ }^{19}$ overcorrection is recommended. ${ }^{24}$ After listening to the voice of the patient and obtaining an optimal result, medialization of the vocal cord $1 \mathrm{~mm}$ more is 
advisable. This is important, because there is always some edema during surgery. If this overcorrection is not done, a small gap will result after the edema is eliminated. This occurred in some of our patients and was clear in the first postoperative assessment ( 1 week postoperatively). So both grade of dysphonia and size of glottic gap were better, though nonsignificant, than the later assessments. This occurs most probably due to the effect of edema, which was absorbed gradually later on. Although edema is absorbed in the late postoperative evaluations, results were still satisfactory until the end of study. The significant difference between the preoperative and postoperative grade of dysphonia revealed the success of the surgery in improving patients' voice. The highly significant difference between preoperative and postoperative gap size revealed the ability of Gore-Tex medialization in approximating the edges of the glottis and eliminating the gap.

In the current study, preoperative acoustic analysis, which is a more objective tool for assessment of voice, could not be assessed due to the large size of the gap, which led to very severe breathy dysphonia. This type of voice could not be detected by our software, although postoperatively the voice signals could be detected and analyzed easily. The comparison between different postoperative results revealed nonsignificant difference. This was evidence of constant improvement of voice signals until the end of the study.

In preoperative assessment, the mean MPT was 7 seconds, which is very short and reveals a great deficiency in the respiratory support for phonation. This was directly due to the large glottic gap, which permitted escape of large amount of air from the chest and larynx. This was corrected after surgery and was clear in the prolongation of MPT (the mean was 20 seconds at the end of the study). The presence of a highly significant difference between preoperative and postoperative MPT confirmed the improvement of the sphincteric mechanism of the glottis. Although the overall improvement of the voice was evident, we cannot deny heterogeneous results of postoperative assessments. This is a logical finding as the initial sample was also heterogeneous.

Piazza et al used type 1 thyroplasty for correction of major GI after endoscopic cordectomy. ${ }^{25}$ When compared with Montgomery Thyroplasty Implant System, they found GoreTex to be easier and more reliable with a smaller extrusion rate. We had only one patient who had endoscopic laser cordectomy for carcinoma in situ. This patient showed significant improvement in his voice quality after Gore-Tex medialization.

The current study demonstrated that the main advantage of Gore-Tex medialization, in addition to the easiness and safety of the procedure, is the excellent and sustained postoperative voice quality. Other advantages include the biocompatibility and safeness of the material itself that was demonstrated by some reports, ${ }^{26,27}$ in which neither an allergic response nor a foreign material reaction were seen during Gore-Tex use. The material is highly compliant and easy to handle. The porous structure of Gore-Tex permits limited cellular infiltration with minimal capsule formation, allowing the material to be isolated from surrounding tissues and easily removed to ensure the reversibility of the surgical procedure. Furthermore, the use of Gore-Tex does not preclude an association with other techniques (e.g., fat injection or anterior commissure laryngoplasty). ${ }^{28}$

\section{Conclusion}

Gore-Tex medialization provides reliable, constant results for both subjective and objective voice parameters. It leads to satisfactory restoration of voice. This technique is relatively easy and does not lead to major complications. Further studies with a larger number of patients and more extended periods of follow-up are still needed to prove the exact efficiency of the technique.

\section{References}

1 Buckmire RA, Bryson PC, Patel MR. Type I Gore-Tex laryngoplasty for glottic incompetence in mobile vocal folds. J Voice 2011;25(3): 288-292

2 Benninger MS, Ahuja AS, Gardner G, Grywalski C. Assessing outcomes for dysphonic patients. J Voice 1998;12(4):540-550

3 Bhattacharyya N, Kotz T, Shapiro J. Dysphagia and aspiration with unilateral vocal cord immobility: incidence, characterization, and response to surgical treatment. Ann Otol Rhinol Laryngol 2002; 111(8):672-679

4 Heitmiller RF, Tseng E, Jones B. Prevalence of aspiration and laryngeal penetration in patients with unilateral vocal fold motion impairment. Dysphagia 2000;15(4):184-187

5 Lam PK, Ho WK, Ng ML, Wei WI. Medialization thyroplasty for cancer-related unilateral vocal fold paralysis. Otolaryngol Head Neck Surg 2007;136(3):440-444

6 Mallur PS, Rosen CA. Vocal fold injection: review of indications, techniques, and materials for augmentation. Clin Exp Otorhinolaryngol 2010;3(4):177-182

7 Spector BC, Netterville JL, Billante C, Clary J, Reinisch L, Smith TL. Quality-of-life assessment in patients with unilateral vocal cord paralysis. Otolaryngol Head Neck Surg 2001;125(3):176-182

8 Dursun G, Boynukalin S, Ozgursoy OB, Coruh I. Long-term results of different treatment modalities for glottic insufficiency. Am J Otolaryngol 2008;29(1):7-12

9 Lu FL, Casiano RR, Lundy DS, Xue JW. Longitudinal evaluation of vocal function after thyroplasty type I in the treatment of unilateral vocal paralysis. Laryngoscope 1996;106(5 Pt 1): 573-577

10 McCulloch TM, Hoffman HT. Medialization laryngoplasty with expanded polytetrafluoroethylene. Surgical technique and preliminary results. Ann Otol Rhinol Laryngol 1998;107(5 Pt 1):427-432

11 Zeitels SM, Mauri M, Dailey SH. Medialization laryngoplasty with Gore-Tex for voice restoration secondary to glottal incompetence: indications and observations. Ann Otol Rhinol Laryngol 2003; 112(2):180-184

12 Soyer T, Lempinen M, Cooper P, Norton L, Eiseman B. A new venous prosthesis. Surgery 1972;72(6):864-872

13 Neel HB III. Implants of Gore-Tex. Arch Otolaryngol 1983;109(7): 427-433

14 Schoenrock LD, Chernoff WG. Subcutaneous implantation of GoreTex for facial reconstruction. Otolaryngol Clin North Am 1995; 28(2):325-340

15 Selber J, Sataloff R, Spiegel J, Heman-Ackah Y. Gore-Tex medialization thyroplasty: objective and subjective evaluation.J Voice 2003; 17(1):88-95

16 Kotby MN. The Accent Method of Voice Therapy. San Diego, CA: Singular Publishing Group, Inc.; 1995 
17 Kotby MN, Bassiouny SE, Youssef M, Abou-elsaad TS. Linear measurements at the vocal fold level. Scientific Journal of ALAzhar Medical Faculty 1995;16:1 (girls)

18 Kotby MN. Voice disorders: recent diagnostic advances. Egypt J Otolaryngol 1986;3(10):69-98

19 Isshiki N, Morita H, Okamura H, Hiramoto M. Thyroplasty as a new phonosurgical technique. Acta Otolaryngol 1974;78(5-6):451-457

20 Montgomery WW, Blaugrund SM, Varvares MA. Thyroplasty: a new approach. Ann Otol Rhinol Laryngol 1993;102(8 Pt 1):571-579

21 Netterville JL, Stone RE, Luken ES, Civantos FJ, Ossoff RH. Silastic medialization and arytenoid adduction: the Vanderbilt experience. A review of 116 phonosurgical procedures. Ann Otol Rhinol Laryngol 1993;102(6):413-424

22 Laccourreye O, Hans S. Endolaryngeal extrusion of expanded polytetrafluoroethylene implant after medialization thyroplasty. Ann Otol Rhinol Laryngol 2003;112(11):962-964

23 Cohen JT, Bates DD, Postma GN. Revision Gore-Tex medialization laryngoplasty. Otolaryngol Head Neck Surg 2004;131(3):236-240
24 Bihari A, Mészáros K, Reményi A, Lichtenberger G. Voice quality improvement after management of unilateral vocal cord paralysis with different techniques. Eur Arch Otorhinolaryngol 2006; 263(12):1115-1120

25 Piazza C, Bolzoni Villaret A, Redaelli De Zinis LO, Cattaneo A, Cocco D, Peretti G. Phonosurgery after endoscopic cordectomies. II. Delayed medialization techniques for major glottic incompetence after total and extended resections. Eur Arch Otorhinolaryngol 2007;264(10):1185-1190

26 Ustundag E, Boyaci Z, Keskin G, Kaur A, Ozkarakas H. Soft tissue response of the larynx to silicone, Gore-Tex, and irradiated cartilage implants. Laryngoscope 2005;115(6):1009-1014

27 Durucu C, Kanlikama M, Mumbuc S, Bayazit Y, Bakir K, Karatas E. Medialization laryngoplasty with Gore-Tex: an animal study. J Voice 2007;21(5):632-639

28 Stasney CR, Beaver ME, Rodriguez M. Minifenestration type I thyroplasty using an expanded polytetrafluoroethylene implant. J Voice 2001;15(1):151-157 